Original article

\title{
Evaluation of the Outcome of Laparoscopic Radical Prostatectomy by a Single Surgeon: Experience with an Initial 30 Cases
}

\author{
Hidetoshi Akita ${ }^{1,3}$, Takehiko Okamura1 ${ }^{1}$ Taku Naiki ${ }^{3}$, Daisuke Nagata ${ }^{2}$, \\ Keiichi Tozawa ${ }^{3}$ and Kenjiro Kohri ${ }^{3}$ \\ ${ }^{1}$ Department of Urology, Anjo Kosei Hospital, Anjo, Japan \\ ${ }^{2}$ Department of Urology, East Medical Center Higashi Municipal Hospital City of Nagoya, Nagoya, Japan \\ ${ }^{3}$ Department of Nephro-urology, Nagoya City University, Graduate School of Medical Science, Nagoya, Japan
}

\begin{abstract}
Objective: We carried out this study to clarify whether operative methods of laparoscopic prostatectomy (LRP) could become a standard therapy. The purpose was to evaluate the technical feasibility, oncologic effectiveness and perioperative and postoperative morbidity of LRP performed by a general urologist. Patients and Methods: Between June 2004 and May 2006, 30 patients with clinically localized prostate cancer consecutively underwent LRP by a single surgeon. Oncologic data were assessed by histopathological examination and by postoperative prostate-specific antigen (PSA) levels.

Results: Complete laparoscopic removal of the prostate and seminal vesicles was achieved in all 30 patients. The average operation time was $250.9 \mathrm{~min}$ (range, 168 to $394 \mathrm{~min}$ ). The total positive surgical margin rate was $20.7 \%$ (6 of 29 cases), with a total PSA recurrence rate was $23.3 \%$ (7 of 30 cases), but the frequencies tended to be decreased in the later phase cases. Perioperative complications were encountered in 5 patients; four of these patients were in the initial 10 cases. Two of the 30 cases (6.7\%) required a blood transfusion (first case and 11th case). There were three surgical complications, one ureter injury, one rectal injury and one sigmoid serosal injury. The catheter duration intervals were reduced in the later cases.

Conclusions: From our experience with one surgeon, with whom perioperative complications were concentrated in the initial 10 cases, we conclude that LRP should be performed by experienced surgeons after intensive training.
\end{abstract}

Key words: laparoscopic radical prostatectomy, prostate cancer

(J Rural Med 2010; 5(1): 134-139)

Correspondence to: Takehiko Okamura

Department of Urology, J.A. Aichi Anjo Kosei Hospital, 28

Higashihirokute, Anjo-cho, Anjo 446-0082, Japan

E-mail: hiko2546@sf.commufa.jp

\section{Introduction}

Early detection of prostate cancer has significantly improved, and a rapid increase in prostate cancer incidence during the past two decades has been noted in many countries $^{1)}$. Radical retropubic prostatectomy (RP) provides excellent long-term disease control for patients with clinically localized prostate cancer but has a negative impact on quality of life ${ }^{2)}$. Laparoscopic surgery is an alternative that is receiving increasing attention. Initially, laparoscopic surgery was used mostly for diagnostic support and for benign lesions, but it is now widely applied for malignancies in many institutions. Between September 1991 and May 1995, Schuessler et al. performed the first series of LRP on nine patients with clinically localized prostate cancer ${ }^{3)}$, and since report of the first 10 cases at two French institutions, this procedure has spread all over the world ${ }^{4,5}$. Several large series of LRP have been reported in France and Germany ${ }^{6,7}$. These investigators demonstrated that LRP can be performed with reasonable operative outcomes and that the approach offers an alternative form of surgical treatment for prostate cancer with reduced patient morbidity. Nowadays, laparoscopic radical cystectomy and urinary diversion using the gastrointestinal tract is routine. Even robotic-assisted laparoscopic surgery is now spreading worldwide. However, laparoscopic procedures are relatively difficult, and it can take considerable time to obtain sufficient experience for a good outcome. In this study, we analyzed the outcome of LRP in initial 30 cases performed by a single surgeon.

\section{Patients and Methods}

Study population

From June 2004 to May 2006, patients with clinically 
Table 1 Clinical and pathologic characteristics of 30 patients

\begin{tabular}{lccc}
\hline Clinical characteristics & Cases 1-10 & Cases 11-20 & Cases 21-30 \\
\hline Mean Age (y) & 66.7 & 67.4 & 62.9 \\
Mean PSA (ng/mL) & 11.4 & 11.7 & 11.7 \\
Gleason score at Biopsy & & & \\
$4-6$ & 4 & 3 & 5 \\
7 & 5 & 3 & 4 \\
$8-10$ & 0 & 3 & 1 \\
Unknown & 1 & 1 & 0 \\
Clinical stage & & & \\
cT1c & 5 & 2 & 4 \\
cT2a,b & 4 & 8 & 6 \\
CT3a & 1 & 0 & 0 \\
\hline
\end{tabular}

There were no significant differences among the three groups.

localized prostate cancer underwent LRP at Nagoya City University Graduate School of Medical Sciences and Anjo Kosei Hospital. From these cases, we retrospectively selected the initial 30 cases receiving this operation performed by a single surgeon, and these cases were then divided into three groups (10 cases each) according to the time period (Groups 1, 2 and 3 included cases 1 to 10, 11 to 20 and 21 to 30, respectively). The average age was 65.7 years (range, 46 to 75 ), and the average preoperative serum PSA level was $11.57 \mathrm{ng} / \mathrm{mL}$ (range, 4 to $22 \mathrm{ng} / \mathrm{mL}$ ). Preoperative staging of the tumor included magnetic resonance imaging, computed tomography and bone scintigraphy. The preoperative clinical stages were as follows: cT1c : cT2a : cT2b $:$ cT3a $=11: 11: 7: 1$. The clinical characteristics are listed in Table 1. There were no significant differences among the three groups. For comparison of peri- and postsurgical parameters among these groups, the oncologic results were evaluated by staging of the operative specimens according to the TNM 1997 classification and according to the last serum PSA level after surgery. The median followup period was 49.7 weeks (range, 17.3 to 87.3 weeks). Histopathologically, the surgical margin in case 28 could not be estimated. We used the Student's $t$ test for comparison of values, with $p<0.05$ considered to be significant.

\section{Investigated parameters}

1. Major complications

2. Operation time

3. Blood loss

4. Catheter indwelling duration after surgery

5. Histopathological tumor stage and surgical margin

6. Postoperative PSA recurrence rates

7. Intervals to pad free and incontinence rates

\section{LRP procedure and histopathological estimation}

All the laparoscopic operative procedures were performed by a single surgeon with 13 years experience in open surgery who received instruction from surgeons with more than 7 years experience in laparoscopic surgery. We were unable to determine who assisted in the procedures because the procedures took place at two separate institutions. The surgical technique was the intraperitoneal approach reported by Guillonneau and Vallancien as the Montsouris technique ${ }^{6}$. In this technique, the patient is positioned at a 30 degree decline in the Trendelenburg position with the pneumoperitoneal pressure maintained at $10 \mathrm{mmHg}$. One 12-mm trocar (blunt tip type) for optical system use is placed under the umbilicus, and another is located inferior to the umbilicus at the lateral border of the right rectus muscle. Three 5-mm trocars are then placed in the lower abdomen, one at the lateral border of the left rectus muscle and one each on the left and right lateral clavicular lines just superior to the anterior superior iliac crest. The surgeon stands to the left of the patient with assistants on the other side. Transperitoneal dissection of the vas deferentia and seminal vesicles is performed through the pouch of Douglas. Denonvilliers' fascia is incised transversely at the base of the prostate, and the bladder is dissected away from the anterior abdominal wall by dividing the median umbilical ligaments and urachus. The Retzius' space is accessed, the endopelvic fascia is incised and Santorini's venous plexus is ligated using 2-0 vicryl on a CTB1 needle (Ethicon). Antegrade prostatectomy begins with anterior bladder neck dissection. With the bladder open, the catheter is grasped, and the prostate is lifted anteriorly to expose the posterior bladder for dissection. The seminal vesicle and vas deferens are grasped to aid in prostatic retraction. Dissection of the lateral pedicles of the prostate, which are selectively coagulated for 
(Min.)

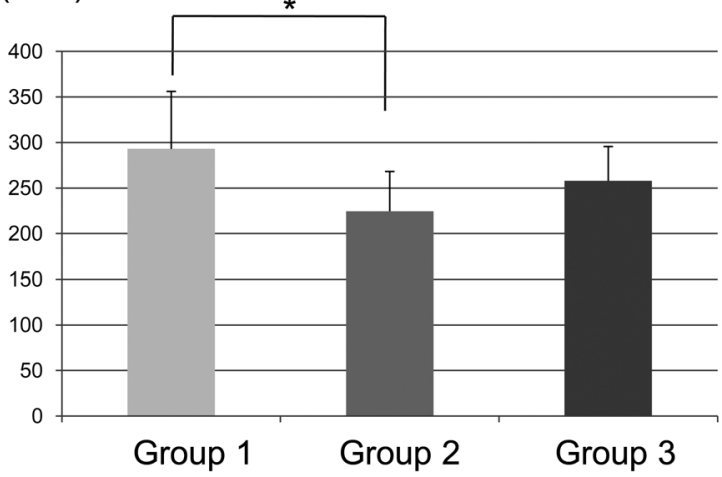

Median
(Min.)

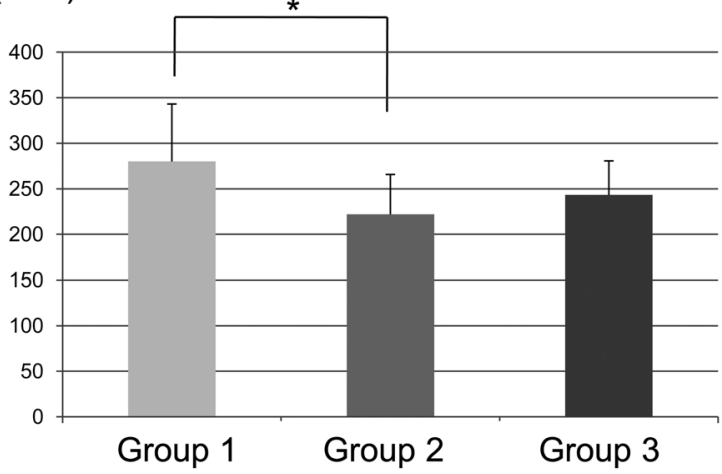

Average

Figure 1 Operation times for the three groups. The difference between Group 1 and Group 2 was statistically significant. $* p<0.05$.

control of bleeding, is performed as far as the apex. Sectioning of the Santorini's plexus is followed by sectioning of the urethra. A vesicourethral anastomosis is performed by running suture with 2-0 vicryl on a UR-6 needle (Ethicon). The final two anterior sutures are placed but not tied until a 20-F Foley catheter has been positioned in the bladder under direct vision. The operative specimen is extracted with a laparoscopy bag from the 12-mm umbilicus trocar. A suction drain is inserted into the retropubic space, and the incisions are closed.

In each case, once the prostate had been removed, the specimen was fixed in $10 \%$ buffered formaldehyde. After removal of the apex and the bladder neck resection margins, the prostate was sectioned axially at regular intervals of 5 $\mathrm{mm}$ or less, yielding serial slices of tissue. On each slide, a pathologist outlined the region of cancer and assigned a Gleason score.

\section{Results}

There was no postoperative mortality, and only one conversion to open surgery was necessary to repair a sigmoid colon serosal injury (10th case). The operation times are described in Figure 1. The average operating times were 280 min (Group 1), 229.5 min (Group 2) and 243.1 min (Group 3). The difference between Group 1 and Group 2 was statistically significant. The operation time relatively decreased as familiarity with the operation increased. The average blood losses were $1498 \mathrm{ml}$ (Group 1), $852.3 \mathrm{ml}$ (Group 2) and $1203 \mathrm{ml}$ (Group 3; Figure 2). Perioperative complications were encountered in 5 patients, four of which were in the initial 10 cases. Two of the 30 cases (6.7\%) required a blood transfusion (first case and 11th case). One patient needed colostomy (8th case) for rectal injury. One patient suffered ureter injury and required ureterostomy (5th case).

The catheter duration after LRP was reduced in the later cases (Figure 3).

The oncologic results are described in Figure 4. The total positive surgical margin rate was $20.7 \%$ (6 of 29 cases), and the total PSA recurrence rate was $23.3 \%$ (7 of the 30 cases). The frequencies tended to be decreased in the late phase group, but without statistical significance.

All patients had their continence status assessed at final follow-up, and $67 \%$ (20 of the 30 cases) were completely continent (no need for a pad). When the recovery period was divided into three groups ( $<3$ months, $\leq 3$ months to $<6$ months, $\leq 6$ months), the number of patients with delayed recovery of continence was slightly increased across the groups, but again without statistical significance (Figure 5).

\section{Discussion}

The majority of LRPs are performed in high volume centers, allowing for intensive learning of complex procedures. In Japan, the procedure is performed by expert urological laparoscopic surgeons, rather than regular urological surgeons, who have expertise in conventional RP. To some extent, our results for our institutions suggest that the laparoscopic approach to radical prostatectomy is a safe and reasonable therapeutic option for treatment of localized prostate cancer. Major complications were concentrated in the initial cases, so that it is important to conduct this operation very carefully under the supervision of teaching staff until a great deal of experience has been obtained. 
(ml)

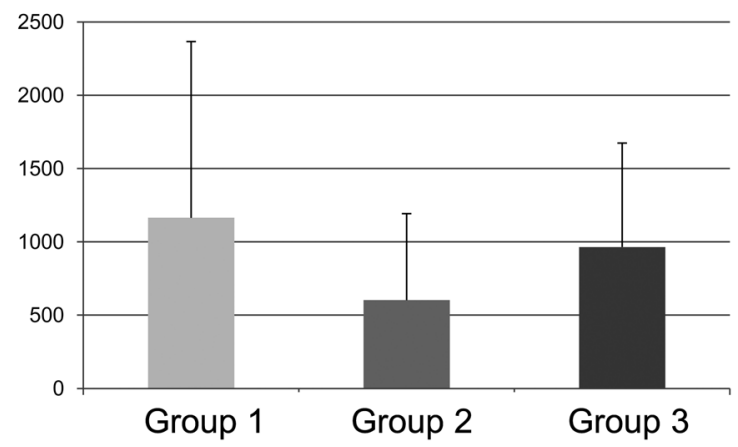

Median (ml)

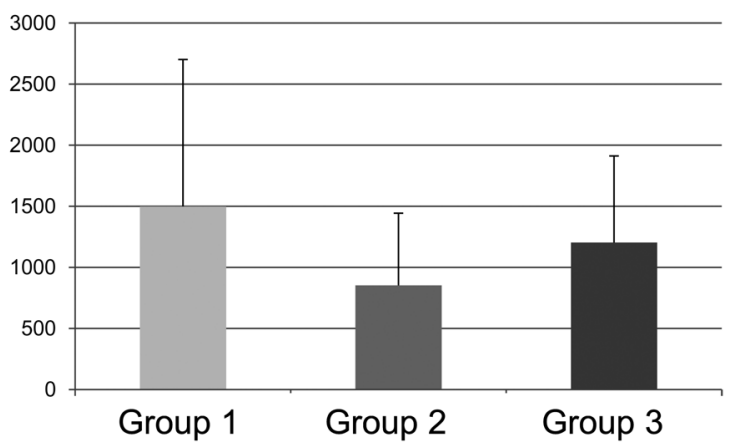

Average

Figure 2 Blood loss with sucked urine. There was no statistical significance among the groups.

(Days)

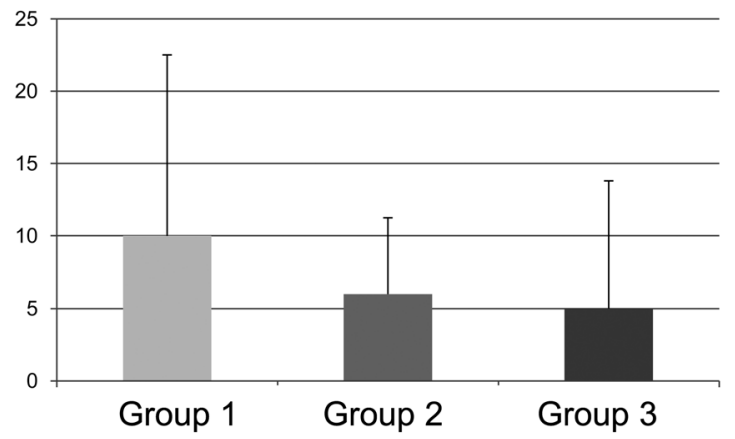

(Days)

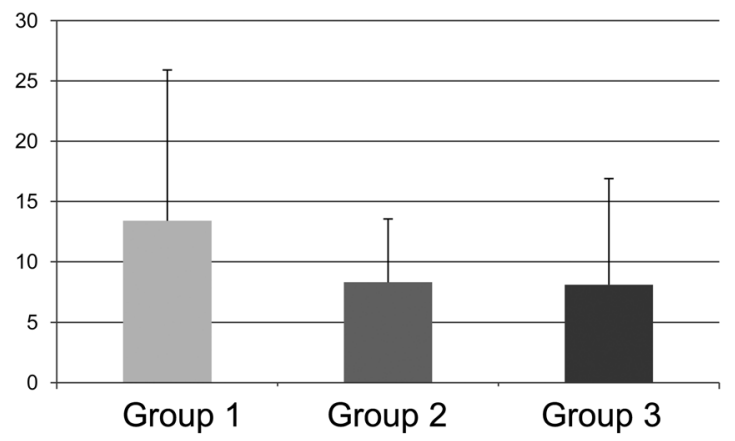

Median

\section{Average}

Figure 3 Catheter duration. The catheter duration after LRP was reduced in the later cases, but there was no statistical significance among the groups.

The post-operative catheter duration, margin positivity rate, and PSA recurrence rate showed a tendency to improve over time, but there was no significant correlation among the three groups. Limited data ${ }^{8}$ are available regarding the initial learning curves for LRP, but Ghavamian et al. ${ }^{9)}$ reported their initial learning curve was overcome after approximately 35 cases, as the mean operation time reached a plateau after that number (mean operation time of 4 hours or less), so we might have to experience further cases. A few reports about the importance of assistants have been published. David et al. ${ }^{10)}$ stressed the importance of one assistant and the placement of ports for maximal benefit in robotic-assisted LRP. Certainly, a single team approach would be optimal, but in our institution this was not always possible.

Continence recovery tended to be relatively late. In postoperative review, this may be a part because of frequent use of monopolar electrocautery for the control of bleeding. As a result, a better view and less positive surgical margin specimens were obtained, but the recovery of continence might thereby have been delayed. We must standardize specific instruments for particular portions of the operation as more experience is gained. Blood loss with sucked urine was worse in Group 3 compared with Group 2. This might be 
Surgical margin

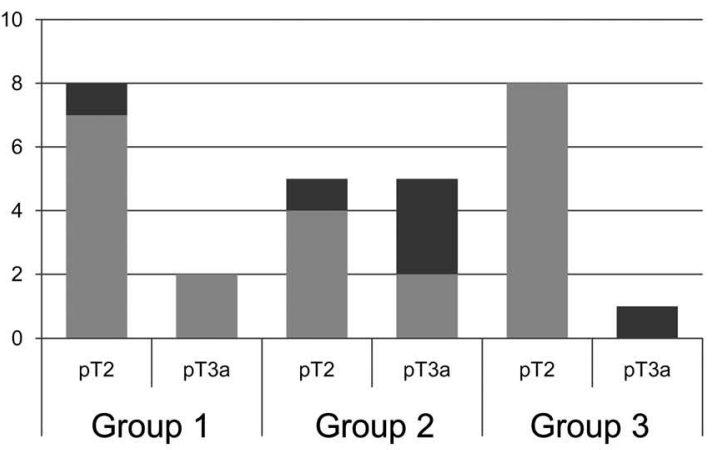

SM (+)

SM (-)
PSA recurrence

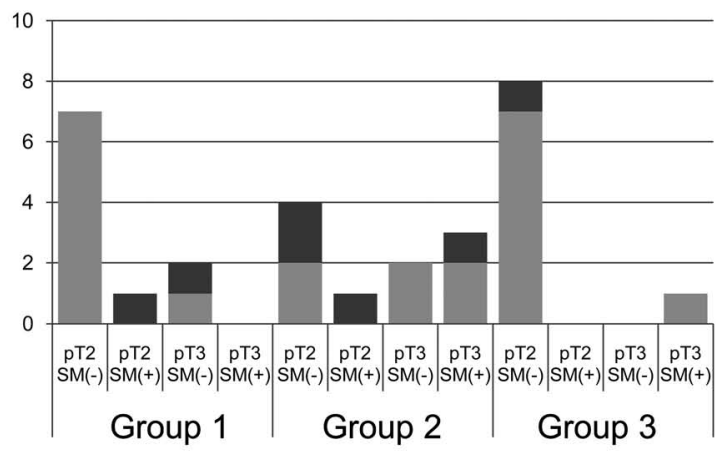

Recurrence (+)

Recurrence (-)

Figure 4 Oncologic results. The frequencies tended to be decreased in the late phase group, but without statistical significance. SM: surgical margin.

$(\%)$

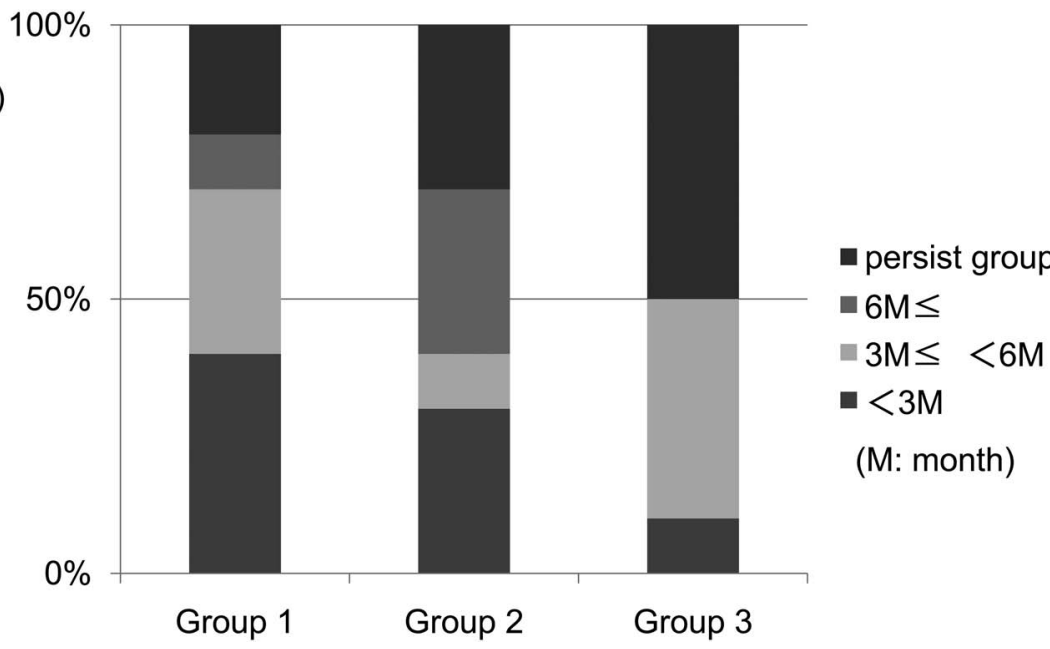

Figure 5 Urinary continence recovery. The number of patients with delayed recovery of continence was slightly increased across the groups, but without statistical significance.

partly due to the presence of different assistants. We might have to pursue a single team, and further investigation is necessary.

From our experience with one surgeon, with whom perioperative complications were concentrated in the initial 10 cases and catheter duration was reduced in the later cases, we conclude that this kind of operation should be performed by experienced surgeons after intensive training.

\section{References}

1. Sarma AV, Schottenfeld D. Prostate cancer incidence, mortality, and survival trends in the United States: 19812001. Semin Urol Oncol 2002; 20: 3-9.

2. Hull GW, Rabbani F, Abbas F, et al. Cancer control with radical prostatectomy alone in 1,000 consecutive patients. J Urol 2002; 167: 528-534.

3. Schuessler WW, Schulman PG, Clayman RV, et al. Laparoscopic radical prostatectomy: Initial short-term 
experience. Urology 1997; 50: 854-857.

4. Jacob F, Salmon L, Hoznek A, et al. Laparoscopic radical prostatectomy: preliminary results. Eur Urol 1999; 37: 615620.

5. Guillonneau B, Cathelineau X, Barret E, et al. Laparoscopic radical prostatectomy: technical and early oncological assessment of 40 operations. Eur Urol 1999; 36: 14-20.

6. Guillonneau B, Vallancien G. Laparoscopic radical prostatectomy: The Montsouris experience. J Urol 2000; 163 : 1643-1649.

7. Rassweiler J, Sentker L, Seemann O, et al. Laparoscopic radical prostatectomy with the Heilbronn technique: an analysis of the first 180 cases. J Urol 2001; 166: 2101-2108.

8. Fabrizio MD, Tuerk I, Schellhammaer PF. Laparoscopic radical prostatectomy: Decreasing the learning curve using a mentor initiated approach. J Urol 2003; 169: 2063-2065.

9. Ghavamian R, Schenk G, Hoenig DM, et al. Overcoming the steep learning curve of laparoscopic radical prostatectomy: single-surgeon experience. J Endourol 2004; 18: 567-571.

10. Lee DI, Eichel L, Skarecky DW, et al. Robotic laparoscopic radical prostatectomy with a single assistant. Urology 2004; 63: $1172-1175$ 